\title{
Bäcklund transformations for discrete Painlevé equations: Discrete $\mathrm{P}_{\mathrm{II}}-\mathrm{P}_{\mathrm{V}}$
}

\author{
A. Sakka ${ }^{\mathrm{a}, *}$, U. Muğan ${ }^{\mathrm{b}}$ \\ a Department of Mathematics, Islamic University of Gaza, P.O. Box 108 Rimal, Gaza, Palestine \\ b Department of Mathematics, Bilkent University, 06800 Bilkent, Ankara, Turkey
}

Accepted 31 March 2005

\begin{abstract}
Transformation properties of discrete Painlevé equations are investigated by using an algorithmic method. This method yields explicit transformations which relates the solutions of discrete Painleve equations, discrete $P_{I I}-P_{V}$, with different values of parameters. The particular solutions which are expressible in terms of the discrete analogue of the classical special functions of discrete Painlevé equations can also be obtained from these transformations.
\end{abstract}

(c) 2005 Elsevier Ltd. All rights reserved.

\section{Introduction}

Painlevé and his school classified the integrable second order equation of the form $y^{\prime \prime}=f\left(x, y, y^{\prime}\right)$ where $f$ is rational in $y$ and $y^{\prime}$ and analytic in $x$, whose solutions have no movable critical points, and discovered six transcendental equations that are called Painlevé equations, $\mathrm{P}_{\mathrm{I}}-\mathrm{P}_{\mathrm{VI}}[1-3]$. Their general solutions cannot be expressed in terms of the known elementary functions and can be regarded as nonlinear analogues of the classical special functions. However, $\mathrm{P}_{\mathrm{II}}-\mathrm{P}_{\mathrm{VI}}$ have rational solutions and solutions expressible in terms of the classical special functions for certain values of parameters. $\mathrm{P}_{\mathrm{II}}-\mathrm{P}_{\mathrm{VI}}$ also possess Bäcklund transformations which relate solutions of the same equation with different values of parameters, or to solution of another equation of Painlevé type [4-6]. Although, Painlevé equations were first discovered from strictly mathematical considerations, they have appeared in physical applications. For example, $\mathrm{P}_{\mathrm{III}}$ arises in the Ising model [7], and $\mathrm{P}_{\mathrm{IV}}$ appears in quantum gravity [8].

Discrete analogues of the Painlevé equations are nonautonomous mappings that are integrable in the same sense as the continues Painlevé equations [9-11], and recently have attracted much attention. The discrete Painlevé equations, d- $\mathrm{P}_{\mathrm{I}}-\mathrm{d}-\mathrm{P}_{\mathrm{VI}}$, which have the form

$$
x_{n+1}=\frac{f_{1}\left(x_{n} ; n\right)+x_{n-1} f_{2}\left(x_{n} ; n\right)}{f_{3}\left(x_{n} ; n\right)+x_{n-1} f_{4}\left(x_{n} ; n\right)},
$$

where $f_{j}\left(x_{n} ; n\right)$ are polynomials of degree at most four in $x_{n}$ [12]. In continuous limit, the discrete Painlevé equations yield a Painlevé equation, though some of the discrete Painlevé equations have limits more then one Painlevé equation.

\footnotetext{
${ }^{*}$ Corresponding author. Fax: +97082863552.

E-mail addresses: asakka@mail.iugaza.edu (A. Sakka), mugan@fen.bilkent.edu.tr (U. Muğan).
} 
Moreover, discrete Painlevé equations possess properties similar to the ones of the continuous Painlevé equations. For example, discrete Painlevé equations also form coalescence cascades [9,13-15], possess Lax's pairs [16-18], have rational solutions for certain values of parameters [19-21], have particular solutions for certain parameter values expressible in terms of the discrete analogue of the special functions [16,19,22-24], and have Bäcklund transformations [19,22,24]. Discrete Painlevé equations also appear in physics, for example, the computation of a certain partition function in a model of two-dimensional quantum gravity led discrete $\mathrm{P}_{\mathrm{I}}$ [25]. The only difference between continuous and discrete Painlevé equations is that the continuous Painleve equations have unique canonical form up to a Möbius transformation, but there is more then one inequivalent discrete equation which has the Painlevé equation as its continuous limit.

Recently, Sakai [26] characterized the Painlevé equations in the frame work of the theory of rational surfaces, and showed that the translation part of the affine Weyl groups give rise to discrete Painlevé equations whereas the whole group acts as their groups of symmetries, Bäcklund transformations. The six continues Painlevé equations appear as degenerate cases of this construction. The geometrical description in the framework of the affine Weyl group $E_{6}^{(1)}$ of the asymmetric $q-\mathrm{P}_{\mathrm{V}}$ and asymmetric $\mathrm{d}-\mathrm{P}_{\mathrm{IV}}$ which are known as discrete analogues of the Painlevé VI equation was given in the recent work of Grammaticos et al. [27].

In this article, we investigate the transformation properties of the discrete Painleve equations by using an algorithmic method which is similar to the method developed by Fokas and Ablowitz [5] for investigating the transformation properties of the continuous equations of the Painlevé type. In [5], for given continuous Painlevé equations

$$
v^{\prime \prime}=P_{2}\left(v^{\prime}\right)^{2}+P_{1} v^{\prime}+P_{3},
$$

where $P_{1}, P_{2}, P_{3}$ depend on $v$, independent variable $z$ and set of parameters $\alpha$, the transformation of type

$$
u(z ; \hat{\alpha})=\frac{v^{\prime}+a v^{2}+b v+c}{d v^{2}+e v+f},
$$

where $a, b, \ldots, d$ depend on $z$ only and $u(z ; \hat{\alpha})$ solves some second order equation of the Painlevé type with set of parameters $\hat{\alpha}$ was considered. If we solve (1.3) for $v^{\prime}$, we obtain

$$
v^{\prime}=(d u-a) v^{2}+(e u-b) v+(f u-c) .
$$

That is, solution $v$ of the given equation of Painleve type also satisfies a Riccati equation with the coefficients depending linearly on the solution $u$ of related Painleve type equation. By following the similar argument, for a given discrete Painlevé Eq. (1.1) with parameter set $\alpha$, we consider discrete Riccati equation, that is

$$
x_{n+1}=\frac{A_{n} x_{n}+B_{n}}{C_{n} x_{n}+D_{n}}
$$

where $A_{n}=A_{1, n} y_{n}+A_{0, n}, B_{n}=B_{1, n} y_{n}+B_{0, n}, C_{n}=C_{1, n} y_{n}+C_{0, n}$, and $D_{n}=D_{1, n} y_{n}+D_{0, n}$ such that $y_{n}$ solves discrete equation of Painlevé type with parameter set $\hat{\alpha}$. The aim is to determine $A_{j, n}, \ldots, D_{j, n}, j=0,1$ requiring that (1.5) defines a one-to-one invertible map between the solutions $x_{n}$ of a given discrete Painlevé equation, and solutions $y_{n}$ of some second order discrete equation of Painlevé type. This method yields explicit transformations between a given discrete Painlevé equation and the same discrete Painlevé equation but with different values of its parameters, and between two different discrete equations of Painlevé type. As an application of the method, we obtain particular solutions of discrete Painlevé equations in terms of discrete analogue of the classical special functions.

The method can be summarized as follows: From Eq. (1.5), one writes

$$
x_{n-1}=-\frac{D_{n-1} x_{n}-B_{n-1}}{C_{n-1} x_{n}-A_{n-1}} .
$$

Substituting $x_{n+1}$ and $x_{n-1}$ given in (1.5) and (1.6) respectively into given discrete Painlevé Eq. (1.1) gives an equation which is polynomial for $x_{n}$ with the coefficients depending on $A_{j, n}, \ldots, D_{j, n}, j=0,1, y_{n}$ and $y_{n-1}$. Now, we choose $A_{j, n}, \ldots, D_{j, n}$ such that the polynomial for $x_{n}$ reduces to a polynomial of degree one or of degree two. That is,

$$
E\left(y_{n}, y_{n-1} ; n\right) x_{n}+F\left(y_{n}, y_{n-1} ; n\right)=0,
$$

or

$$
E\left(y_{n}, y_{n-1} ; n\right) x_{n}^{2}+F\left(y_{n}, y_{n-1} ; n\right) x_{n}+G\left(y_{n}, y_{n-1} ; n\right)=0 .
$$

If one solves (1.7) or (1.8) for $x_{n}$ and substitutes into (1.5), (1.5) yields a discrete equation of Painlevé type for $y_{n}$. It turns out that, similar to the case of continuous Painlevé equations, discrete $\mathrm{P}_{\mathrm{II}}-\mathrm{P}_{\mathrm{V}}$ admit transformations of both types (1.7) and (1.8). However, discrete $\mathrm{P}_{\mathrm{VI}}$ does not admit a transformation of type (1.7). In this article we will consider the transformation of type (1.7), the type (1.8) will be considered later and published elsewhere. 


\section{Discrete Painlevé II equation}

In this section, we consider d-P $\mathrm{PII}_{\text {equation: }}$

$$
x_{n+1}+x_{n-1}=\frac{z_{n} x_{n}+a}{1-x_{n}^{2}},
$$

where $z_{n}=\alpha n+\beta$, and $\alpha, \beta, a$ are constants. Substituting $x_{n+1}$ and $x_{n-1}$ given in (1.5) and (1.6) respectively into (2.1) gives the following polynomial for $x_{n}$,

$$
\left(z_{n} x_{n}+a\right)\left(C_{n} x_{n}+D_{n}\right)\left(C_{n-1} x_{n}-A_{n-1}\right)=\left(1-x_{n}^{2}\right)\left[\left(A_{n} x_{n}+B_{n}\right)\left(C_{n-1} x_{n}-A_{n-1}\right)-\left(C_{n} x_{n}+D_{n}\right)\left(D_{n-1} x_{n}-B_{n-1}\right)\right] .
$$

Our goal now is to choose $A_{n}, \ldots, D_{n}$ in such a way that (2.2) becomes a linear equation for $x_{n}$. Eq. (2.2) reduces to a linear equation for $x_{n}$,

$$
\left(B_{n}+B_{n-1}+z_{n}-2\right) x_{n}=B_{n}-B_{n-1}-a,
$$

with the choice of $A_{n}=C_{n}=D_{n}=1$. Without loss of generality, we may choose $B_{n}=y_{n}-\frac{1}{2} z_{n}-\frac{1}{4} \alpha+1$. Hence, Eqs. (1.5) and (2.3) respectively give

$$
y_{n}=\left(x_{n}+1\right)\left(x_{n+1}-1\right)+\frac{1}{2} z_{n}+\frac{1}{4} \alpha
$$

and

$$
x_{n}=\frac{y_{n}-y_{n-1}+v}{y_{n}+y_{n-1}},
$$

where $v=-\frac{1}{2} \alpha-a$. Eliminating $x_{n}$ between (2.4) and (2.5) leads to a discrete form of $\mathrm{P}_{\mathrm{XXXIV}}$ [22]:

$$
\left(y_{n}+y_{n-1}\right)\left(y_{n}+y_{n+1}\right)=\frac{v^{2}-4 y_{n}^{2}}{y_{n}-\frac{1}{2} z_{n}-\frac{1}{4} \alpha} .
$$

Miura transformation (2.5) was also given in [22].

\subsection{Bäcklund transformation for $d-P_{I I}$}

Since, (2.6) is quadratic in $v$, thus $y_{n}(v)=y_{n}(-v)$. But then, from Eq. (2.5)

$$
\bar{x}_{n}(-v)=\frac{y_{n}(-v)-y_{n-1}(-v)-v}{y_{n}(-v)+y_{n-1}(-v)}=\frac{y_{n}(v)-y_{n-1}(v)-v}{y_{n}(v)+y_{n-1}(v)}=x_{n}(v)-\frac{2 v}{y_{n}(v)+y_{n-1}(v)} .
$$

Hence, expressing $y_{n}$ in terms of $x_{n}$ and using $v=-\frac{1}{2} \alpha-a$ give the following Bäcklund transformation for d- $\mathrm{P}_{\mathrm{II}}$

$$
\bar{x}_{n}=x_{n}-\frac{2 v\left(x_{n}+1\right)}{2\left(x_{n+1}-1\right)\left(x_{n}+1\right)-z_{n} x_{n}-a} ; \quad \bar{a}=-a-\alpha .
$$

Bäcklund transformation for d-P $\mathrm{P}_{\text {II }}$ was also given in $[22,24,28]$.

\subsection{Special solution}

The transformation (2.5) breaks down if

$$
y_{n}+y_{n-1}=0
$$

and

$$
y_{n}-y_{n-1}+v=0 .
$$

By solving (2.9) and (2.10), we find that $y_{n}=v=0$. Substituting $y_{n}=v=0$ into (2.4) yields the following discrete Riccati equation:

$$
x_{n+1}=\frac{2 x_{n}-z_{n}+a+2}{2\left(x_{n}+1\right)} .
$$


Therefore, particular solution of d- $\mathrm{P}_{\mathrm{II}}$ is characterized by (2.11), iff $a=-\frac{1}{2} \alpha$. Eq. (2.11) can be linearized by a ColeHopf transformation $x_{n}=\frac{w_{n}}{w_{n-1}}-1$, and we thus obtain the discrete analogue of the Airy equation:

$$
2 w_{n+1}+\left(z_{n}-a\right) w_{n-1}-4 w_{n}=0 .
$$

Special solution in terms of discrete Airy functions of d-P $\mathrm{P}_{\mathrm{II}}$ was also given in [22-24].

\section{Discrete Painlevé III equation}

In this section, we consider the following discrete Painlevé III equation, q-P $\mathrm{P}_{\text {III }}$ [14]:

$$
x_{n+1} x_{n-1}=\frac{a b\left(x_{n}-c \lambda^{n}\right)\left(x_{n}-d \lambda^{n}\right)}{\left(x_{n}-a\right)\left(x_{n}-b\right)},
$$

where $a, b, c, d$ are constants. Using the method introduced in Section 1, we find

$$
\frac{\left(A_{n} x_{n}+B_{n}\right)\left(D_{n-1} x_{n}-B_{n-1}\right)}{\left(C_{n} x_{n}+D_{n}\right)\left(C_{n-1} x_{n}-A_{n-1}\right)}=-\frac{a b\left(x_{n}-c \lambda^{n}\right)\left(x_{n}-d \lambda^{n}\right)}{\left(x_{n}-a\right)\left(x_{n}-b\right)} .
$$

With the choice of $D_{n}=-a C_{n}$ and $A_{n}=b C_{n}$, Eq. (3.2) can be reduced to the following linear equation for $x_{n}$ :

$$
\left[b C_{n} B_{n-1}+a C_{n-1} B_{n}+a b(c+d) \lambda^{2 n} C_{n} C_{n-1}\right] x_{n}=a b c d \lambda^{2 n} C_{n} C_{n-1}-B_{n} B_{n-1} .
$$

Without loss of generality we may let, $B_{n}=\mu \lambda^{n+\frac{1}{2}} y_{n}, C_{n}=1$, where $\mu^{2}=a b c d$. Then Eqs. (1.5) and (3.3) become

$$
y_{n}=\frac{x_{n+1}\left(x_{n}-a\right)-b x_{n}}{\mu \lambda^{n+\frac{1}{2}}}
$$

and

$$
x_{n}=\frac{\mu^{2} \lambda^{n+\frac{1}{2}}\left(1-y_{n} y_{n-1}\right)}{a \mu \lambda y_{n}+b \mu y_{n-1}+a b \lambda^{\frac{1}{2}}(c+d)},
$$

respectively. Eliminating $x_{n}$ between the Eqs. (3.4) and (3.5) leads to the following discrete equation for $y_{n}$

$$
\left(y_{n} y_{n+1}-1\right)\left(y_{n} y_{n-1}-1\right)=-\frac{\bar{\lambda}^{n}\left(y_{n}-\alpha\right)\left(y_{n}-\frac{1}{\alpha}\right)\left(y_{n}-\beta\right)\left(y_{n}-\frac{1}{\beta}\right)}{\left(\gamma y_{n}-\bar{\lambda}^{n}\right)}
$$

where

$$
\alpha=-\frac{\mu}{a c \lambda^{\frac{1}{2}}}, \quad \beta=-\frac{\mu}{a d \lambda^{\frac{1}{2}}}, \quad \gamma=-\frac{\mu \lambda^{\frac{1}{2}}}{a b}, \quad \bar{\lambda}=\lambda^{-1} .
$$

Eq. (3.6) is the special case, $\delta=0$, of q- $\mathrm{P}_{\mathrm{V}}[14]$ :

$$
\left(y_{n} y_{n+1}-1\right)\left(y_{n} y_{n-1}-1\right)=\frac{\bar{\lambda}^{2 n}\left(y_{n}-\alpha\right)\left(y_{n}-\frac{1}{\alpha}\right)\left(y_{n}-\beta\right)\left(y_{n}-\frac{1}{\beta}\right)}{\left(\gamma y_{n}-\bar{\lambda}^{n}\right)\left(\delta y_{n}-\bar{\lambda}^{n}\right)} .
$$

Thus there exists the one to one correspondence given by (3.4) and (3.5) between the solutions of q-P $\mathrm{P}_{\text {III }}$ and (3.6).

\subsection{Bäcklund transformation for discrete $P_{I I I}$}

Bäcklund transformation can be obtained by finding two sets of $\{\alpha, \beta, \gamma\}$ such that (3.6) is invariant. It should be noted that Eq. (3.6) is invariant under the change of parameters

$$
\bar{\alpha}=\frac{1}{\alpha}, \quad \bar{\beta}=\frac{1}{\beta}, \quad \bar{\gamma}=\gamma .
$$

By using (3.9) and following the same procedure given in Section 2.1, we obtain the following Bäcklund transformation for $\mathrm{q}-\mathrm{P}_{\mathrm{III}}$ 


$$
\begin{aligned}
& \bar{x}_{n}=\frac{\bar{d} \lambda x_{n}\left[a x_{n+1}\left(x_{n}-a\right)+b x_{n-1}\left(x_{n}-b\right)-2 a b x_{n}+a b(c+d) \lambda^{n}\right]}{c\left[b x_{n+1}\left(x_{n}-a\right)+a \lambda^{2} x_{n-1}\left(x_{n}-b\right)-\left(a^{2} \lambda^{2}+b^{2}\right) x_{n}+a b(c+d) \lambda^{n+1}\right]} \\
& \bar{a}=\frac{b \bar{d}}{\lambda c}, \quad \bar{b}=\frac{a \lambda \bar{d}}{c}, \quad \bar{c}=\frac{d \bar{d}}{c}
\end{aligned}
$$

\subsection{Special solution}

The transformation (3.5) breaks down if

$$
y_{n} y_{n-1}-1=0
$$

and

$$
a \mu \lambda y_{n}+b \mu y_{n-1}+a b \lambda^{\frac{1}{2}}(c+d)=0 .
$$

By solving Eqs. (3.11) and (3.12), we find that $y_{n}=-\frac{b c}{\mu \sqrt{\lambda}}$, and $\lambda \mu^{2}=b^{2} c^{2}$. Then, (3.4) leads to the following discrete Riccati equation

$$
x_{n+1}=\frac{b\left(x_{n}-c \lambda^{n}\right)}{x_{n}-a} .
$$

Therefore, particular solution of $q-\mathrm{P}_{\mathrm{III}}$ is characterized by (3.13), iff $\lambda a d=b c$. Eq. (2.11) can be linearized by a ColeHopf transformation $x_{n}=a+\frac{w_{n}}{w_{n-1}}$, and we thus obtain the discrete analogue of the Bessel equation [16]:

$$
w_{n+1}-b\left(a-c \lambda^{n}\right) w_{n-1}+(a-b) w_{n}=0 .
$$

The linearizability condition for q-P $\mathrm{P}_{\mathrm{III}}$ and the particular solution expressible in terms of the discrete analogue of the Bessel functions were also obtained in [15,16,29].

\section{Discrete Painlevé IV equation}

In this section, we consider the discrete Painlevé IV equation, $\mathrm{d}-\mathrm{P}_{\mathrm{IV}}$ :

$$
\left(x_{n+1}+x_{n}\right)\left(x_{n-1}+x_{n}\right)=\frac{\left(x_{n}^{2}-a^{2}\right)\left(x_{n}^{2}-b^{2}\right)}{\left(x_{n}-z_{n}\right)^{2}-c^{2}},
$$

where $z_{n}=\alpha n+\beta$, and $a, b, \alpha, \beta$ are constants. Eq. (4.1) gives the following equation after substituting $x_{n+1}$ and $x_{n-1}$ respectively given in (1.5) and (1.6),

$$
\frac{\left[C_{n} x_{n}^{2}+\left(D_{n}+A_{n}\right) x_{n}+B_{n}\right]\left[C_{n-1} x_{n}^{2}-\left(D_{n-1}+A_{n-1}\right) x_{n}+B_{n-1}\right]}{\left(C_{n} x_{n}+D_{n}\right)\left(C_{n-1} x_{n}-A_{n-1}\right)}=\frac{\left(x_{n}^{2}-a^{2}\right)\left(x_{n}^{2}-b^{2}\right)}{\left(x_{n}-z_{n}\right)^{2}-c^{2}} .
$$

Eq. (4.2) can be reduced to a linear equation for $x_{n}$

$$
x_{n}=\frac{-\left(y_{n-1}-z_{n}+\mu+\alpha\right)\left(y_{n}-z_{n}-\mu-\alpha\right)+z_{n}^{2}-c^{2}}{y_{n}+y_{n-1}},
$$

with the choice of $A_{n}=-\left(y_{n}-z_{n}+\mu\right), B_{n}=a b, C_{n}=1$, and $D_{n}=y_{n}-z_{n}-\mu-\alpha$, where $\mu=-\frac{1}{2}(a+b+\alpha)$. With these choices, Eq. (1.5) yields

$$
x_{n+1}=\frac{-\left(y_{n}-z_{n}+\mu\right) x_{n}+a b}{x_{n}+y_{n}-z_{n}-\mu-\alpha} .
$$

By eliminating $x_{n}$ between (4.3) and (4.4), we obtain d-P $\mathrm{IV}$

$$
\left(y_{n+1}+y_{n}\right)\left(y_{n-1}+y_{n}\right)=\frac{\left(y_{n}^{2}-\bar{a}^{2}\right)\left(y_{n}^{2}-\bar{b}^{2}\right)}{\left(y_{n}-\overline{z_{n}}\right)^{2}-\bar{c}^{2}} \text {, }
$$

where

$$
\bar{z}_{n}=z_{n}+\frac{1}{2} \alpha, \quad \bar{a}^{2}=\left[c-\frac{1}{2}(a+b-\alpha)\right]^{2}, \quad \bar{b}^{2}=\left[c+\frac{1}{2}(a+b-\alpha)\right]^{2}, \quad \bar{c}^{2}=\frac{1}{4}(a-b)^{2} .
$$


If we replace $y_{n}$ with $\bar{x}_{n}$ in (4.4), we thus obtain the following Bäcklund transformation for d-P $\mathrm{IV}$

$$
\bar{x}_{n}=-\frac{\left[x_{n+1}\left(x_{n}-z_{n}-\mu-\alpha\right)-x_{n}\left(z_{n}-\mu\right)-a b\right]}{\left(x_{n+1}+x_{n}\right)},
$$

such that $\bar{x}_{n}$ solves d-P $\mathrm{P}_{\mathrm{IV}}$ with the parameters $\bar{a}, \bar{b}, \bar{c}$ given by (4.6). The Bäcklund transformation (4.7) for discrete Painlevé IV equation was first given in [19].

\subsection{Special solution}

The transformation (4.3) breaks down if

$$
y_{n}+y_{n-1}=0
$$

and

$$
-\left(y_{n-1}-z+\mu+\alpha\right)\left(y_{n}-z_{n}-\mu-\alpha\right)+z^{2}-c^{2}=0 .
$$

$y_{n}=\mu+\alpha+c=0$ solve Eqs. (4.8) and (4.9). Eq. (4.4) yields the following discrete Riccati equation,

$$
x_{n+1}=\frac{\left(a+b-c+z_{n}\right) x_{n}+a b}{x_{n}+c-z_{n}},
$$

after substituting $y_{n}=\mu+\alpha+c=0$ [15,19,29]. Therefore, particular solution of d-P IV satisfies (4.10), iff $a+b-2 c=\alpha$. Cole-Hopf transformation $x_{n}=z_{n}-c+\left(w_{n} / w_{n-1}\right)$ transforms the Riccati equation (4.10) into the following linear equation for $w_{n}$ :

$$
w_{n+1}-\left(z_{n}-c+a\right)\left(z_{n}-c+b\right) w_{n-1}-2 c w_{n}=0 .
$$

Eq. (4.11) has been shown to be solvable in terms of the discrete analogues of Hermite functions [19].

\section{Discrete Painlevé V equation}

In this section, we consider the $\mathrm{q}-\mathrm{P}_{\mathrm{V}}$ equation

$$
\left(x_{n} x_{n+1}-1\right)\left(x_{n} x_{n-1}-1\right)=\frac{\lambda^{2 n}\left(x_{n}-a\right)\left(x_{n}-b\right)\left(x_{n}-\frac{1}{a}\right)\left(x_{n}-\frac{1}{b}\right)}{\left(c x_{n}-\lambda^{n}\right)\left(d x_{n}-\lambda^{n}\right)},
$$

where $a, b, c, d$ are constants. Applying the method introduced in Section 1, we find

$$
\frac{\left[A_{n} x_{n}^{2}+\left(B_{n}-C_{n}\right) x_{n}-D_{n}\right]\left[-D_{n-1} x_{n}^{2}+\left(B_{n-1}-c_{n-1}\right) x_{n}+A_{n-1}\right]}{\left(C_{n} x_{n}+D_{n}\right)\left(C_{n-1} x_{n}-A_{n-1}\right)}=\frac{\lambda^{2 n}\left(x_{n}-a\right)\left(x_{n}-b\right)\left(x_{n}-\frac{1}{a}\right)\left(x_{n}-\frac{1}{b}\right)}{\left(c x_{n}-\lambda^{n}\right)\left(d x_{n}-\lambda^{n}\right)} .
$$

Eq. (5.2) can be reduced to a linear equation for $x_{n}$ with the choice of $A_{n}=\mu \lambda^{n}, B_{n}=y_{n}-(a+b) \mu \lambda^{n}, C_{n}=y_{n}$ and $D_{n}=-a b A_{n}$, where $\mu^{2}=\frac{\lambda}{a b c d}$. Then Eqs. (5.2) and (1.5) yield

$$
x_{n}=\frac{\mu \lambda^{n-1}\left(a b \lambda y_{n-1}+y_{n}\right)-\left(\frac{1}{c}+\frac{1}{d}\right) \lambda^{n}}{y_{n} y_{n-1}-1}
$$

and

$$
x_{n+1}=\frac{\mu \lambda^{n}\left(x_{n}-a-b\right)+y_{n}}{y_{n} x_{n}-a b \mu \lambda^{n}},
$$

respectively. We obtain the following q- $\mathrm{P}_{\mathrm{V}}$ for $y_{n}$ by eliminating $x_{n}$ between Eqs. (5.3) and (5.4):

$$
\left(y_{n} y_{n+1}-1\right)\left(y_{n} y_{n-1}-1\right)=\frac{\lambda^{2 n}\left(y_{n}-\alpha\right)\left(y_{n}-\frac{1}{\alpha}\right)\left(y_{n}-\beta\right)\left(y_{n}-\frac{1}{\beta}\right)}{\left(\gamma y_{n}-\lambda^{n}\right)\left(\delta y_{n}-\lambda^{n}\right)},
$$


where $\gamma=\frac{1}{a \mu}, \delta=\frac{1}{b \mu}, \alpha=\frac{\mu c}{\lambda}$, and $\beta=\frac{\mu d}{\lambda}$. Therefore, we have the following Bäcklund transformation for q-P $\mathrm{P}_{\mathrm{V}}$

$$
\begin{aligned}
& \bar{x}_{n}=\frac{\mu \lambda^{n}\left[a b x_{n+1}+x_{n}-a-b\right]}{x_{n} x_{n+1}-1} ; \\
& \bar{a}=\frac{\mu c}{\lambda}, \quad \bar{b}=\frac{\mu d}{\lambda}, \quad \bar{c}=\frac{1}{a \mu}, \quad \bar{d}=\frac{1}{b \mu} .
\end{aligned}
$$

\subsection{Special solution}

The transformation (5.3) breaks down if

$$
y_{n} y_{n-1}-1=0
$$

and

$$
\mu \lambda^{n-1}\left(a b \lambda y_{n-1}+y_{n}\right)-\left(\frac{1}{c}+\frac{1}{d}\right) \lambda^{n}=0 .
$$

If we substitute the solutions $y_{n}=\frac{\lambda}{\mu c}$, and $\lambda^{2}=\mu^{2} c^{2}$ of (5.7) and (5.8) into (5.4), we obtain the following discrete Riccati equation [29],

$$
x_{n+1}=\frac{\lambda^{n}\left(x_{n}-a-b\right)+a b d}{a b\left(d x_{n}-\lambda^{n}\right)} .
$$

Therefore, particular solution of q- $\mathrm{P}_{\mathrm{V}}$ is characterized by (5.9), iff $c=\lambda a b d$. Eq. (2.11) can be linearized by a Cole-Hopf transformation $x_{n}=\frac{\lambda^{n}}{d}\left(1-\frac{w_{n}}{w_{n-1}}\right)$, and we thus obtain the following linear equation for $w_{n}$

$$
w_{n+1}-\frac{1}{\lambda}\left(\frac{1}{a}-\frac{d}{\lambda^{n}}\right)\left(\frac{1}{b}-\frac{d}{\lambda^{n}}\right) w_{n-1}+\left(\frac{1}{a b \lambda}-1\right) w_{n}=0 .
$$

The linearization condition of $\mathrm{q}-\mathrm{P}_{\mathrm{V}}$ was also given in [30], and shown that $x_{n}$ can be expressed in terms of discrete analogue of confluent hypergeometric functions.

\section{Conclusion}

In this article, we have presented an algorithm which is similar to the algorithm introduced in [5] for continuous Painlevé equations, to obtain the Bäcklund transformations for discrete $\mathrm{P}_{\mathrm{II}}-\mathrm{P}_{\mathrm{V}}$. The algorithm is simple and based on the investigation of discrete Riccati Eq. (1.5) for the solution of a given discrete Painlevé equation, with the coefficients depending linearly on the solution of another discrete equation of Painlevé type. The Miura transformation

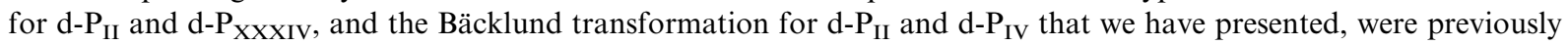
known. The special-function solutions for the discrete Painlevé equations are extensively covered in the literature. But the transformations for $\mathrm{q}-\mathrm{P}_{\mathrm{III}}$ and $\mathrm{q}-\mathrm{P}_{\mathrm{V}}$ were not discussed in the literature before. Moreover, as an application of the algorithm, we have presented the special solutions which are discrete analogue of the classical special functions, for discrete $\mathrm{P}_{\mathrm{II}}-\mathrm{P}_{\mathrm{V}}$ whenever the parameters satisfy certain conditions (the linearizability conditions).

\section{Acknowledgement}

This work was partially supported by the Scientific and Technical Research Council of Turkey (TÜBITAK).

\section{References}

[1] Painlevé P. Acta Math 1902;25:1.

[2] Gambier B. Acta Math 1909;33:1.

[3] Ince EL. Ordinary differential equations. New York: Dover; 1956.

[4] Airault H. Stud Appl Math 1979;61:31-53.

[5] Fokas AS, Ablowitz MJ. J Math Phys 1982;23:2033-42.

[6] Gromak VI, Laine I, Shimomura S. Painlevé differential equations in the complex plane. Berlin, New York: Walter de Gruyter; 2002. 
[7] McCoy BM, Perk JHH, Shrock RE. Nucl Phys B 1983;220:35-47.

[8] Fokas AS, Its AR, Kitaev AV. Commun Math Phys 1991;142:313-44.

[9] Ramani A, Grammaticos B, Hietarinta J. Phys Rev Lett 1991;67:1829-32.

[10] Grammaticos B, Nijhoff FW, Ramani A. Discrete Painlevé equations. In: Conte R, editor. The Painlevé Propert, One century Later. CRM series in mathematical physics. New York: Springer; 1999. p. 413-516.

[11] Grammaticos B, Ramani A. Meth Appl Ann 1991;4:1829.

[12] Quispel GRW, Roberts JAG, Thompson CJ. Physica D 1989;34:183.

[13] Grammaticos B, Ramani A. Discrete Painlevé equations: derivations and properties. NATO ASI C 1993;413:299.

[14] Ramani A, Grammaticos B. Physica A 1996;228:160-71. solv-int/9510011.

[15] Tamizhmani KM, Ramani A, Grammaticos B, Kajiwara K. J Phys A: Math Gen 1998;31:5799-810.

[16] Grammaticos B, Nijhoff FW, Papageorgiou V, Ramani A, Satsuma J. Phys Lett A 1994;185:446-52. solv-int/9310003.

[17] Papageorgiou VG, Nijhoff FW, Grammaticos B, Ramani A. Phys Lett A 1992;164:57-64.

[18] Joshi N, Bartoclay D, Halburd RG. Lett Math Phys 1992;26:123.

[19] Tamizhmani KM, Grammaticos B, Ramani A. Lett Math Phys 1993;29:49-54.

[20] J. Hietarinta, K. Kajiwara, Rational solutions to d-P $\mathrm{I}$, solv-int/9705002.

[21] Kajiwara K, Yamamoto K, Ohta Y. Phys Lett A 1997;232:189-99.

[22] Ramani A, Grammaticos B. J Phys A: Math Gen 1992;25:L633-7.

[23] Kajiwara K, Ohta Y, Satsuma J, Grammaticos B, Ramani A. J Phys A: Math Gen 1994;27:915-22.

[24] Carstea AS, Ramani A, Willox R, Grammaticos B. J Phys A: Math Gen 2003;36:8419-31.

[25] Brezin E, Kazakov V. Phys Lett B 1990;236:144-50.

[26] Sakai H. Commun Math Phys 2001;220:165-229.

[27] Grammaticos B, Ramani A, Ohta Y. J Non Math Phys 2003;10:215-28.

[28] Muğan U, Sakka A, Santini PM. Phys Lett A 2005;336:37-45.

[29] Tamizhmani T, Grammaticos B, Ramani A, Tamizhmani KM. Physica A 2001;295:359.

[30] Tamizhmani KM, Grammaticos B, Ramani A, Ohta Y. Lett Math Phys 1996;38:289. 